\title{
Optimization of plasmon excitation at structured apertures
}

\author{
Evgeny Popov, Michel Nevière, Anne-Laure Fehrembach, and Nicolas Bonod
}

\begin{abstract}
Surface plasmon excitation that is due to a single or a structured circular aperture in a flat metallic screen is investigated theoretically and numerically with a view to enhancing the electric field close to the metallic surface. A systematic study of the homogeneous solution of the electromagnetic scattering problem is made with cylindrical coordinates, expanding Maxwell equations on a Fourier-Bessel basis. A perturbation analysis devoted to simple physical analyses of different types of cylindrical nanostructure is developed for the optimization of plasmon excitation by a normally incident linearly polarized monochromatic plane wave. The conclusions drawn from this analysis agree well with the results of rigorous electromagnetic calculations obtained with the differential theory of diffraction in cylindrical coordinates. (C) 2005 Optical Society of America

OCIS codes: $240.6680,050.1220$.
\end{abstract}

\section{Introduction}

The discovery of the so-called extraordinary light transmission through subwavelength hole arrays $^{1}$ triggered an avalanche of studies with interest extending far beyond the physics community. Recent biophysical applications used single apertures as nanocavities in trapping single biomolecules and in their investigation. Field enhancement inside the nanostructures is aimed at further reducing the investigated volume, as well as for use in near-field microscopy applications, which may require using a coaxial intrusion inside the aperture ${ }^{2}$ or structuring its neighborhood by surface corrugation. ${ }^{3,4}$

There exist many numerical studies of periodic hole arrays, based mainly on grating theories, but only few have been devoted to isolated single (or structured) apertures in real metals. The precision of finite-difference time-domain methods is restricted by the small dimensions of the structures compared with the wavelength, requiring special approaches. This method has been used with success for elliptical

E. Popov (e.popov@fresnel.fr), M. Nevière, and A.-L. Fehrembach are with the Institut Fresnel, Unité Mixte de Recherche Associée au Centre National de la Recherche Scientifique 6133, Université de Provence, Faculté des Sciences et Techniques de St. Jérôme, Avenue Escadrille Normandie Niémen, 13397 Marseille Cedex 20, France. N. Bonod is with Commissariat à l'Energie Atomique, Centre d'Etudes Scientifiques et Techniques d'Aquitaine, B.P. 2, 33114 Le Barp, France.

Received 22 March 2005; revised manuscript received 9 May 2005; accepted 9 May 2005.

0003-6935/05/296141-14\$15.00/0

(C) 2005 Optical Society of America apertures by application of local grid refinement. 5 Recently a differential theory of diffraction in cylindrical geometry that can be used to model cylindrical objects of finite length was developed. ${ }^{6}$ Its application to single-hole diffraction ${ }^{7}$ demonstrated the key role played by surface plasmons in a near-field distribution, even in the absence of periodicity.

Our aims in the present paper are to study in detail the specific character of a plasmon-type homogeneous solution of the diffraction problem in cylindrical symmetry, to develop a perturbation analysis of single and structured apertures, to compare the analytical conclusions with rigorous electromagnetic computations, and to use them for maximizing the plasmon excitation. The structure under consideration is schematically represented in Fig. 1. It consists of a single circular aperture surrounded by concentric lamellar grooves of circular form. The grooves are assumed to match the phase of the incident wave to the plasmon surface wave to enhance it, an expectation well motivated by numerous effects related to resonance anomalies in diffraction gratings. However, it is not clear what geometry is necessary for this phase matching when cylindrical symmetry exists. Using a direct analogy with grating structures, one would expect that the grooves should be equidistant. However, such is not the case, as we show below.

Figure 2 illustrates the spectral dependence of the $z$ component of the plasmon wave on a $230 \mathrm{~nm}$ thick silver screen, calculated outside the corrugated region (at $x=4 \mu \mathrm{m}$ ) for four $30 \mathrm{~nm}$ deep channels situated about a circular aperture with a $50 \mathrm{~nm}$ radius (the second curve, representing the spectral behavior of coupling integral $F_{0}$, is discussed further in 


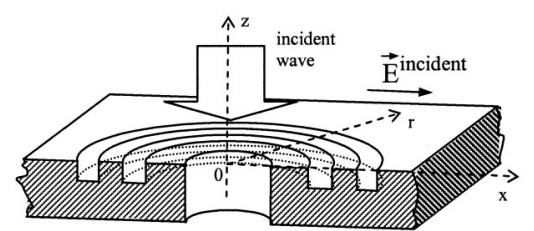

(a)

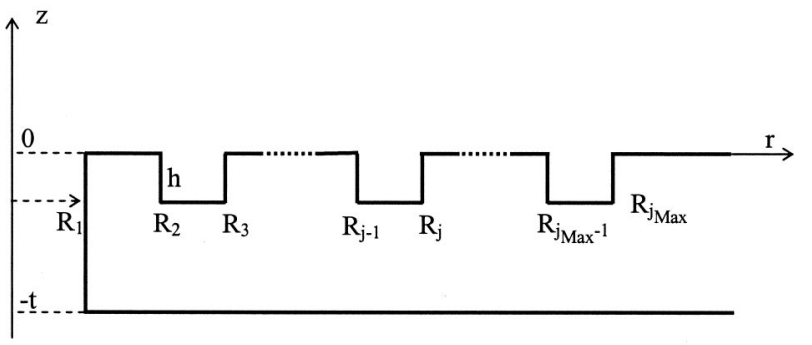

(b)

Fig. 1. Schematic representation of a structured aperture: (a) general view, (b) cross section with the notation used in the text.

the text). The channel walls are equidistant and have radii $R_{j}=(j-1) \times 235 \mathrm{~nm}(j=2, \ldots 9)$, which corresponds to a quasi-period of $470 \mathrm{~nm}$. These values are chosen in order that the aperture will have a radius approximately ten times smaller than the optimum wavelength, a ratio that is of practical importance in biomolecular studies and near-field microscopy. According to expectations from the onedimensional grating theories, this geometry has to resonantly (in-phase) excite a plasmon surface wave that has propagation constant $\alpha^{p} \approx \lambda / d$. When one takes into account the refractive index of silver, the spectral maximum has to be close to $500 \mathrm{~nm}$. However, instead of a maximum, one finds a minimum. In what follows we shall try first to explain this phenomenon and second to present a simple physical method that will enable us to optimize plasmon excitation in cylindrical geometry. To this end we need first to discuss the diffraction of light and plasmon propagation in cylindrical geometry. Although these

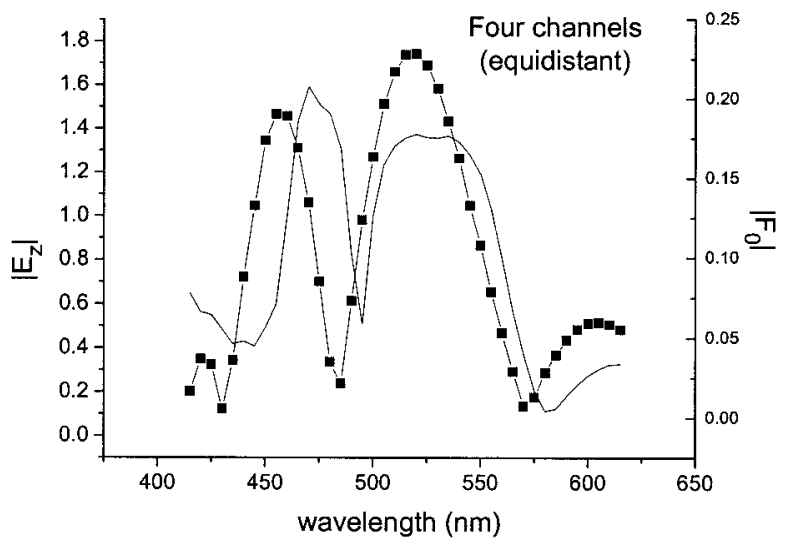

Fig. 2. Spectral dependence of $\left|E_{z}\right|$ calculated at a point $(4 \mu \mathrm{m}, 0,0)$ for a silver film with $t=230 \mathrm{~nm}$ thickness, $R_{1}$ $=50 \mathrm{~nm}$, four equidistant channels with $h=30 \mathrm{~nm}$, and $\lambda$ $=500 \mathrm{~nm}$. topics are not new, ${ }^{8-11}$ we find this necessary in order to use the results in formulating a perturbative approach to a physical explanation of numerical results presented in Section 5 below.

\section{Propagation Equations and Their Solutions in Cylindrical Coordinates}

Because of the natural $2 \pi$ periodicity with respect to $\theta$, the electric field vectorial components can be represented by Fourier series in $\theta$ :

$$
E_{j}(r, \theta, z)=\sum_{n=-\infty}^{+\infty} E_{j, n}(r, z) \exp (i n \theta), \quad j=r, \theta, z,
$$

and a similar expression could be written for magnetic field $H$. In what follows, we assume that dielectric permittivity $\varepsilon$ is piecewise constant in $z$ and independent of $\theta$. This independence ensures that Maxwell equations and boundary conditions are decoupled in $\theta$, i.e., that the propagation and scattering of each field component $E_{j, n}$ are carried out separately and independently for each value of $n$. Thus the three-dimensional electromagnetic problem is reduced to $N_{n}$ two-dimensional problems, where $N_{n}$ is the number of terms retained when the series in Eq. (1) is truncated during the calculations.

Helmholtz equations in cylindrical coordinates (see Appendix A) lead to coupling between $E_{r, n}$ and $E_{\theta, n}$ :

$$
\begin{aligned}
& \Delta E_{\theta, n}-\frac{E_{\theta, n}}{r^{2}}+\frac{2 i n}{r^{2}} E_{r, n}+k^{2} E_{\theta, n}=0, \\
& \Delta E_{r, n}-\frac{E_{r, n}}{r^{2}}-\frac{2 i n}{r^{2}} E_{\theta, n}+k^{2} E_{r, n}=0,
\end{aligned}
$$

where $k=\omega \sqrt{\mu_{0} \varepsilon}$ is the wave number and the Laplacian takes the form

$$
\Delta=\frac{\partial^{2}}{\partial r^{2}}+\frac{1}{r} \frac{\partial}{\partial r}+\frac{1}{r^{2}} \frac{\partial^{2}}{\partial \theta^{2}}+\frac{\partial^{2}}{\partial z^{2}} .
$$

In what follows, $k_{0}$ will denote the free-space wave number.

By making two linear combinations of $E_{r, n}$ and $E_{\theta, n}$ it is possible to decouple Eqs. (2). Defining

$$
E_{\bar{\mp}, n}=E_{\theta, n} \pm i E_{r, n}
$$

yields for Eqs. (2)

$$
\left[\frac{\partial^{2}}{\partial r^{2}}+\frac{1}{r} \frac{\partial}{\partial r}-\frac{(n \pm 1)^{2}}{r^{2}}+k^{2}+\frac{\partial^{2}}{\partial z^{2}}\right] E_{ \pm, n}(r, z)=0 .
$$

Separating variables $r$ and $z$ makes it easy to identify here the differential equations that generate Bessel functions of order $n \pm 1$, which are denoted hence- 
forth by $\mathbb{Z}_{n+1}$. An infinite unperturbed plane interface requires a nondiverging solution for each $r$, which limits the choice to first-kind Bessel functions $J_{n \pm 1}\left(k_{r} r\right)$, where $k_{r}$ is a free parameter serving to separate the variables in Eq. (5). It will appear later that $k_{r}$ represents the radial component of the wave vector.

As we discuss below, a surface perturbation could act as a localized source for a plasmon surface wave such that other Bessel functions could serve as a solution and, in particular, the Hankel function $\mathbf{H}_{n \pm 1}^{+}\left(k_{r} r\right)$, which represents a wave propagating in a radial direction away from the source toward $r \rightarrow \infty$. Because of the completeness of the Bessel-function basis, the general solution of Eq. (5) can be written as a linear combination of particular solutions obtained for each value of $k_{r}$ :

$$
E_{ \pm, n}(r, z)=\int_{0}^{\infty} e_{ \pm, n}\left(k_{r}, z\right) \mathbb{Z}_{n \pm 1}\left(k_{r} r\right) k_{r} \mathrm{~d} k_{r} .
$$

Equation (5) determines the $z$ variation of the field components:

$$
\int_{0}^{\infty} \mathbb{Z}_{n \pm 1}\left(k_{r} r\right) k_{r} \mathrm{~d} k_{r}\left(k_{r}^{2}-k^{2}-\frac{\partial^{2}}{\partial z^{2}}\right) e_{ \pm, n}\left(k_{r}, z\right)=0 .
$$

When one considers a homogeneous region with permittivity independent of $r$, too, and uses the orthogonality of the Bessel functions of the same order with respect to their arguments, Eq. (5) is further decoupled with respect to $k_{r}$. To observe this it is necessary to multiply both sides of Eq. (7) by $\mathbb{Z}_{n \pm 1}\left(k_{r} r\right) r$ and to integrate with respect to $r$ from zero to infinity. Recalling that

$$
\int_{0}^{\infty} \mathbb{Z}_{n \pm 1}\left(k_{r}^{\prime} r\right) \mathbb{Z}_{n \pm 1}\left(k_{r} r\right) r \mathrm{~d} r=\frac{\delta\left(k_{r}^{\prime}-k_{r}\right)}{k_{r}^{\prime}},
$$

with $\delta$ representing the Dirac function, we obtain

$$
-\frac{\partial^{2}}{\partial z^{2}} e_{ \pm, n}\left(k_{r}, z\right)=\left(k^{2}-k_{r}^{2}\right) e_{ \pm, n}\left(k_{r}, z\right) .
$$

Obviously, the solutions represent a combination of waves propagating upward and downward along the $z$ axis:

$$
\begin{aligned}
& e_{+, n}^{ \pm}\left(k_{r}, z\right)=b_{n}^{E, \pm}\left(k_{r}\right) \exp \left( \pm i k_{z} z\right), \\
& e_{-, n}^{ \pm}\left(k_{r}, z\right)=c_{n}^{E, \pm}\left(k_{r}\right) \exp \left( \pm i k_{z} z\right),
\end{aligned}
$$

with

$$
k_{z}=\sqrt{k^{2}-k_{r}^{2}} .
$$

Equations (10) and (11) establish that $k_{r}$ is the radial component of the wave vector. In what follows, the superscripts + and - refer to the upgoing and the downgoing elementary waves, respectively.

The same formulas can be written for the magnetic field components by use of the notation $h$ instead of $e$ and $H$ instead of $E$. Thus our new set of parameters for each value of $k_{r}$ will consist of eight coefficients, $b_{n}^{E, \pm}, b_{n}^{H, \pm}, c_{n}^{E, \pm}$, and $c_{n}^{H, \pm}$. They can serve to express the remaining $z$ components of the electromagnetic field by using Maxwell equations:

$$
\begin{aligned}
i \omega \mu_{0} H_{z} & =(\operatorname{rot} \vec{E})_{z}, \\
i \omega \varepsilon E_{z} & =-(\operatorname{rot} \vec{H})_{z} .
\end{aligned}
$$

After some trivial calculations, one can obtain expressions similar to Eqs. (6) and (10):

$$
\begin{aligned}
H_{z, n}(r, z)= & \frac{1}{i \omega \mu_{0}} \int_{0}^{\infty} \mathbb{Z}_{n}\left(k_{r} r\right) k_{r} \mathrm{~d} k_{r} \\
& \times\left[b_{n}^{E,{ }^{ \pm}}\left(k_{r}\right)-c_{n}^{E,{ }^{ \pm}}\left(k_{r}\right)\right] \exp \left( \pm i k_{z} z\right), \\
E_{z, n}(r, z)= & -\frac{1}{i \omega \varepsilon} \int_{0}^{\infty} \mathbb{Z}_{n}\left(k_{r} r\right) k_{r} \mathrm{~d} k_{r} \\
& \times\left[b_{n}^{H, \pm}\left(k_{r}\right)-c_{n}^{H,{ }^{ \pm}}\left(k_{r}\right)\right] \exp \left( \pm i k_{z} z\right) .
\end{aligned}
$$

The form of these expressions indicates that it is useful to consider two different polarizations with respect to the $z$ axis: (i) transverse electric (TE) polarization with respect to the $z$ axis, for which $E_{z}=0$, which implies that $b_{n}^{H, \pm}=c_{n}^{H, \pm}$, and (ii) transverse magnetic (TM) polarization with respect to the $z$ axis, for which $H_{z}=0$. This implies that $b_{n}^{E, \pm}=c_{n}^{E, \pm}$.

Maxwell's equations (Appendix A) lead to further links between the field components, so each polarization is characterized by

TE polarization:

$$
\begin{aligned}
& b_{n}^{H, \pm}=c_{n}^{H, \pm}=\frac{ \pm i k_{z}}{\omega \mu_{0}} b_{n}^{E, \pm}, \\
& b_{n}^{E, \pm}=-c_{n}^{E, \pm} ;
\end{aligned}
$$

TM polarization:

$$
\begin{aligned}
& b_{n}^{E, \pm}=c_{n}^{E, \pm}=\frac{\mp i k_{z}}{\omega \varepsilon} b_{n}^{H, \pm}, \\
& b_{n}^{H, \pm}=-c_{n}^{H, \pm} .
\end{aligned}
$$

Thus for each polarization a single independent couple of parameters represents upgoing and downgoing waves.

\section{Reflection on a Plane Interface and Surface Plasmon Wave in Cylindrical Coordinates}

Let us consider plane interface(s) perpendicular to the $z$ axis between different media characterized by 
different values of $\varepsilon$. It is well known that the tangential component of the incident wave vector is preserved when the interfaces are crossed. In cylindrical coordinates, this is radial component $k_{r}$. Whereas this rule in Cartesian coordinates is quite familiar from university courses, one can easily get convinced of its validity by applying the procedure of Eq. (8) to the field expansions in cylindrical coordinates at each side of the interface and by using the orthogonality of Bessel functions.

Thus each elementary solution with a given value of $k_{r}$ is independent of the solutions for other values of $k_{r}$. The plane interface couples only the upgoing and downgoing component for each $k_{r}$. However, such is not the case for an interface with apertures, which will couple all $k_{r}$ components, thus leading to diffraction phenomena.

Equations (13) show that the fundamental TE and TM polarizations are preserved at each interface, because of the continuity of $\mu_{0} H_{z}$ and $\varepsilon E_{z}$. Thus, as is done in Cartesian coordinates, an arbitrary polarized incident field can be decomposed into two fundamental polarizations, and the reflected and transmitted fields will be the sum of the fields reflected and transmitted in each polarization, independently of the other polarization. Let us consider the two cases, taking for simplicity a single interface at $z=0$ that separates the upper medium with permittivity $\hat{\varepsilon}$ from the lower medium with permittivity $\varepsilon$.

We denote by $\hat{b}_{n}^{E,-}$ and $\hat{b}_{n}^{H,-}$ the TE and TM waves incident from the upper medium; the continuity of the tangential electric and magnetic field components implies, from Eqs. (6) and (10) and the analogous equations for $H$, that tesian coordinates. The only possible surface waves exist in TM polarization when the interface separates a metal from a dielectric. There is nothing surprising in obtaining the same results when one is working in cylindrical coordinates, for the following reasons: A plane-wave plasmon solution in Cartesian coordinates is characterized by its TM nature with respect to the plane of propagation; i.e., the magnetic field vector is parallel to the interface, independently of the direction of propagation along this interface. Thus any combination of plasmons propagating in different directions will have $H_{z}$ $=0$. They all will have a unique propagation constant $k_{r}^{p}$ along the radial direction, equal to the pole of the TM reflection and transmission Fresnel coefficients, a solution of the equation

$$
\frac{\left[\hat{k}^{2}-\left(k_{r}^{p}\right)^{2}\right]^{1 / 2}}{\hat{\varepsilon}}+\frac{\left[k^{2}-\left(k_{r}^{p}\right)^{2}\right]^{1 / 2}}{\varepsilon}=0,
$$

where $\hat{k}^{2}=\omega^{2} \mu_{0} \hat{\varepsilon}$. The corresponding $z$ components of the wave vector are given by

$$
\begin{aligned}
& \hat{k}_{z}^{p}=\left[\hat{k}^{2}-\left(k_{r}^{p}\right)^{2}\right]^{1 / 2}, \\
& k_{z}^{p}=\left[k^{2}-\left(k_{r}^{p}\right)^{2}\right]^{1 / 2},
\end{aligned}
$$

with nonnegative imaginary parts.

From Eqs. (4), (6), (10), (15), and (16), the electric field of the elementary plasmon surface wave in cylindrical coordinates can be derived:

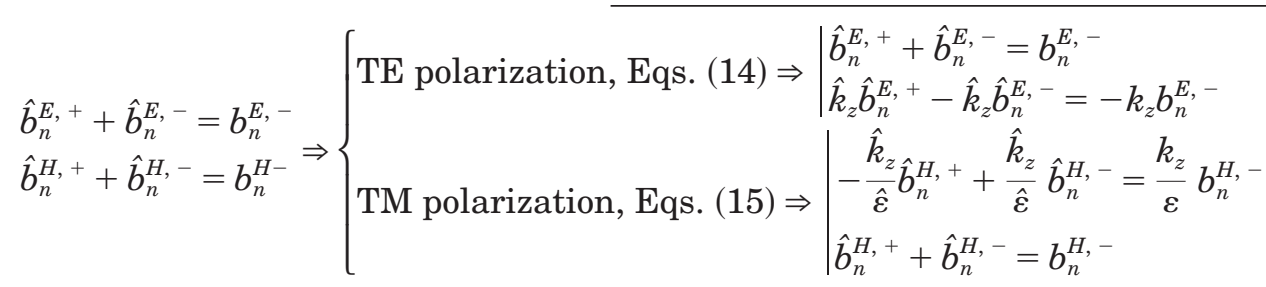

Thus the Fresnel coefficients attain the same form ${ }^{8}$ as in Cartesian coordinates:

$$
\begin{aligned}
r^{\mathrm{TE}} \equiv \frac{\hat{b}_{n}^{E,+}}{\hat{b}_{n}^{E,-}}=\frac{\hat{k}_{z}-k_{z}}{\hat{k}_{z}+k_{z}}, & r^{\mathrm{TM}} \equiv \frac{\hat{b}_{n}^{H,+}}{\hat{b}_{n}^{H,-}}=\frac{\hat{k}_{z} / \hat{\varepsilon}-k_{z} / \varepsilon}{\hat{k}_{z} / \hat{\varepsilon}+k_{z} / \varepsilon}, \\
t^{\mathrm{TE}} \equiv \frac{b_{n}^{E,-}}{\hat{b}_{n}^{E,-}}=\frac{2 \hat{k}_{z}}{\hat{k}_{z}+k_{z}}, & t^{\mathrm{TM}} \equiv \frac{b_{n}^{H,-}}{\hat{b}_{n}^{H,-}}=\frac{2 \hat{k}_{z} / \hat{\varepsilon}}{\hat{k}_{z} / \hat{\varepsilon}+k_{z} / \varepsilon} .
\end{aligned}
$$

As one can observe from Eqs. (18), nonmagnetic media cannot exhibit TE polarized surface waves, ${ }^{10,11}$ a fact that is well known when one is working in Car-

$$
\begin{aligned}
\hat{E}_{r, n, k_{r}^{p}}^{p}(r, \theta, z)= & \frac{-i}{2}\left(\hat{E}_{-, n, k_{r}^{p}}^{p}-\hat{E}_{+, n, k_{r}^{p}}^{p}\right) \exp (i n \theta) \\
= & \frac{\hat{k}_{z}^{p}}{2 \omega \hat{\varepsilon}} \hat{b}_{n}^{H,+}\left(\hat{k}_{r}^{p}\right) \exp \left(i n \theta+i \hat{k}_{z}^{p} z\right) \\
& \times\left[\mathbb{Z}_{n-1}\left(\hat{k}_{r}^{p} r\right)-\mathbb{Z}_{n+1}\left(k_{r}^{p} r\right)\right], \\
\hat{E}_{\theta, n, k_{r}^{p}}^{p}(r, \theta, z)= & \frac{1}{2}\left(\hat{E}_{-, n, k_{r}^{p}}^{p}+\hat{E}_{+, n, k_{r}^{p}}^{p}\right) \exp (i n \theta) \\
= & \frac{i \hat{k}_{z}^{p}}{2 \omega \hat{\varepsilon}} \hat{b}_{n}^{H,+}\left(k_{r}^{p}\right) \exp \left(i n \theta+i \hat{k}_{z}^{p} z\right) \\
& \times\left[\mathbb{Z}_{n-1}\left(k_{r}^{p} r\right)+\mathbb{Z}_{n+1}\left(k_{r}^{p} r\right)\right],
\end{aligned}
$$




$$
\hat{E}_{z, n, k_{r}^{p}}^{p}(r, \theta, z)=\frac{2 i k_{r}^{p}}{\omega \hat{\varepsilon}} \hat{b}_{n}^{H,+}\left(k_{r}^{p}\right) \exp \left(i n \theta+i \hat{k}_{z}^{p} z\right) \mathbb{Z}_{n}\left(k_{r}^{p} r\right)
$$

In addition, Eq. (19) ensures the continuity of $E_{r}, E_{\theta}$, and $\varepsilon E_{z}$. In the lower medium the field has the same form, except that we have to suppress the circumflex and replace $\hat{k}_{z}^{p}$ with $-k_{z}^{p}$. Let us consider the field forms that correspond to several values of $n$ and have different degrees of symmetry.

\section{A. $n=0$}

The elementary field that corresponds to $n=0$ is $\theta$ independent. It has no $\theta$ electric field component $\left(J_{-1}=-J_{1}\right)$ and represents a radial plasmon that propagates from the origin in all radial directions. The $r$ dependence is given by $J_{0}\left(k_{r}^{p} r\right)$ for the $z$ component and by $J_{1}\left(k_{r}^{p} r\right)$ for the $r$ component. Such a surface wave is quite difficult to excite, because it requires a radially polarized source field.

\section{B. $n= \pm 1$}

The most important elementary solution regarding excitation with a linearly polarized incident field, the most common experimental situation when one is using laser sources, is $n= \pm 1$. From this point of view it is more important to consider two independent linear combinations that include $n=-1$ and $n=1$, although they could be treated separately. Let us consider two combinations, the sum $(p+)$ and the difference $(p-)$, between the components for $n$ $=-1$ and $n=+1$ that have the following properties (it is necessary to remember that each solution of the homogeneous problem is determined within a multiplicative constant):

1. $\hat{b}_{-1}^{H,+}=\hat{b}_{+1}^{H,+}$

$$
\begin{aligned}
\hat{E}_{r, p+, k_{r}^{p}}^{p}(r, \theta, z)= & \frac{i \hat{k}_{z}^{p}}{\omega \hat{\varepsilon}} \hat{b}_{1}^{H,+}\left(k_{r}^{p}\right) \exp \left(i \hat{k}_{z}^{p} z\right) \\
& \times\left[\mathbb{Z}_{0}\left(k_{r}^{p} r\right)-\mathbb{Z}_{2}\left(k_{r}^{p} r\right)\right] \sin (\theta),
\end{aligned}
$$

$\hat{E}_{\theta, p+, k_{r}^{p}}^{p}(r, \theta, z)=\frac{i \hat{k}_{z}^{p}}{\omega \hat{\varepsilon}} \hat{b}_{1}^{H,+}\left(k_{r}^{p}\right) \exp \left(i \hat{k}_{z}^{p} z\right)$

$$
\times\left[\mathbb{Z}_{0}\left(k_{r}^{p} r\right)+\mathbb{Z}_{2}\left(k_{r}^{p} r\right) 0\right] \cos (\theta),
$$

$\hat{E}_{z, p+, k_{r}^{p}}^{p}(r, \theta, z)=\frac{-4 \hat{k}_{r}^{p}}{\omega \hat{\varepsilon}} \hat{b}_{1}^{H,+}\left(k_{r}^{p}\right) \exp \left(i \hat{k}_{z}^{p} z\right) \mathbb{Z}_{1}\left(k_{r}^{p} r\right) \sin (\theta)$.

These equations can be further simplified by use of the relations between Bessel functions:

$$
\begin{aligned}
\hat{E}_{r, p+, k_{r}^{p}}^{p}(r, \theta, z)= & \frac{2 i \hat{k}_{z}^{p}}{\omega \hat{\varepsilon}} \hat{b}_{1}^{H,+}\left(k_{r}^{p}\right) \exp \left(i \hat{k}_{z}^{p} z\right) \mathbb{Z}_{1}^{\prime}\left(k_{r}^{p} r\right) \sin (\theta), \\
\hat{E}_{\theta, p+, k_{r}^{p}}^{p}(r, \theta, z)= & \frac{i \hat{k}_{z}^{p}}{\omega \hat{\varepsilon}} \hat{b}_{1}^{H,+}\left(k_{r}^{p}\right) \\
& \times \exp \left(i \hat{k}_{z}^{p} z\right) \frac{2}{k_{r}^{p} r} \mathbb{Z}_{1}\left(k_{r}^{p} r\right) \cos (\theta), \\
\hat{E}_{z, p+, k_{r}^{p}}^{p}(r, \theta, z)= & \frac{-4 \hat{k}_{r}^{p}}{\omega \hat{\varepsilon}} \hat{b}_{1}^{H,+}\left(k_{r}^{p}\right) \\
& \times \exp \left(i \hat{k}_{z}^{p} z\right) \mathbb{Z}_{1}\left(k_{r}^{p} r\right) \sin (\theta),
\end{aligned}
$$

which form gives a better understanding of the field behavior. Recalling the asymptotic behavior of Bessel functions [see relations (24) below], one can observe first that the $\theta$ component of the field declines as $\exp \left(\operatorname{Im} k_{r}^{p} r\right) / r^{3 / 2}$, much faster than the $r$ and $z$ components, which behave asymptotically as $\exp \left(\operatorname{Im} k_{r}^{p} r\right) / r^{1 / 2}$.

Second, the $r$ and $z$ components are zero at $\theta$ $=0, \pi$; thus the electric field declines much more rapidly in directions close to $\theta=0, \pi$ and much more slowly in directions $\theta= \pm(\pi / 2)$, as if the plasmon were able to propagate more easily along the $y$ axis than along the $x$ axis. In addition, along the $y$ axis, component $E_{\theta} \equiv \pm E_{x}$ is null, as happens for a plane plasmon wave propagating along $y$.

Third, $E_{z}$ and $E_{r}$ are antisymmetric with respect to the directions of propagation, i.e., when $\theta$ changes its sign.

Fourth, depending on the type of solution $\left(J_{n}\right.$ or $\mathbf{H}_{n}^{+}$), the modulus of each component decreases with $r$ but either varies rapidly, representing a standingwave feature (for $J_{n}$ ), or represents a wave propagating toward $r \rightarrow \infty$ (for $\mathbf{H}_{n}^{+}$), which could be observed in their asymptotic behavior:

$$
\begin{gathered}
J_{1}\left(k_{r}^{p} r\right) \underset{r \rightarrow \infty}{\rightarrow} \sqrt{2 / \pi k_{r}^{p} r} \cos \left(k_{r}^{p} r-\frac{3}{4} \pi\right), \\
\mathbf{H}_{n}^{+}\left(k_{r}^{p} r\right) \underset{r \rightarrow \infty}{\rightarrow} \sqrt{2 / \pi k_{r}^{p} r} \exp \left(i k_{r}^{p} r-i \frac{3}{4} \pi\right) .
\end{gathered}
$$

2. $\hat{b}_{-1}^{H,+}=-\hat{b}_{+1}^{H,+}$

Equations (21) are simplified to take the form

$$
\begin{aligned}
\hat{E}_{r, p-, k_{r}^{p}}^{p}(r, \theta, z)= & \frac{2 \hat{k}_{z}^{p}}{\omega \hat{\varepsilon}} k_{r}^{p} \hat{b}_{1}^{H,+}\left(k_{r}^{p}\right) \exp \left(i \hat{k}_{z}^{p} z\right) \mathbb{Z}_{1}^{\prime}\left(k_{r}^{p} r\right) \cos (\theta), \\
\hat{E}_{\theta, p-, k_{r}^{p}}^{p}(r, \theta, z)= & \frac{-\hat{k}_{z}^{p}}{\omega \hat{\varepsilon}} k_{r}^{p} \hat{b}_{1}^{H,+}\left(k_{r}^{p}\right) \exp \left(i \hat{k}_{z}^{p} z\right) \frac{2}{k_{r}^{p} r} \mathbb{Z}_{1}\left(k_{r}^{p} r\right) \\
& \times \sin (\theta), \\
\hat{E}_{z, p-, k_{r}^{p}}^{p}(r, \theta, z)= & \frac{4 i\left(k_{r}^{p}\right)^{2}}{\omega \hat{\varepsilon}} \hat{b}_{1}^{H,+}\left(k_{r}^{p}\right) \exp \left(i \hat{k}_{z}^{p} z\right) \mathbb{Z}_{1}\left(k_{r}^{p} r\right) \cos (\theta),
\end{aligned}
$$


quite similar to Eqs. (23); the only difference is as if $\theta \rightarrow \theta+(\pi / 2)$. The same conclusions are valid as when $\hat{b}_{-1}^{H,+}=\hat{b}_{+1}^{H,+}$ with axes $x$ and $y$ interchanged.

C. $\mathrm{n}= \pm 2, \pm 3, \ldots$

Higher-order solutions can easily be obtained from the general form written in Eqs. (21). Similarly to the case with $n= \pm 1$, the solutions can be combined to

$$
\hat{b}_{n}^{H,-}\left(k_{r}\right)=k_{0}^{2} \hat{b}_{n}^{H, i} \frac{\delta\left(k_{r}-k_{r}^{i}\right)}{k_{r}^{i}} .
$$

Let us recall that we are interested in TM polarization because a plasmon wave does not exist in TE polarization. Because of the coupling between the diffracted components, the simple equation (16) is replaced by a more complicated form:

$$
\mid \begin{aligned}
& \frac{1}{\hat{\varepsilon}} \int_{0}^{\infty} \hat{k}_{z}\left(k_{r}\right) \exp \left(i \hat{k}_{z} z_{s}\right) \hat{b}_{n}^{H,+}\left(k_{r}\right) \mathbb{Z}_{n+1}\left(k_{r} r\right) k_{r} \mathrm{~d} k_{r}-k_{0}^{2} \frac{\hat{k}_{z}^{i}}{\hat{\varepsilon}} \exp \left(-i \hat{k}_{z}^{i} z_{s}\right) \hat{b}_{n}^{H, i} J_{n+1}\left(k_{r}^{i} r\right)=-\frac{1}{\varepsilon} \int_{0}^{\infty} k_{z}\left(k_{r}\right) \exp \left(-i k_{z} z_{s}\right) b_{n}^{H,-}\left(k_{r}\right) \mathbb{Z}_{n+1}\left(k_{r} r\right) k_{r} \mathrm{~d} k_{r}, \\
& \int_{0}^{\infty} \exp \left(i \hat{k}_{z} z_{s}\right) \hat{b}_{n}^{H,+}\left(k_{r}\right) \mathbb{Z}_{n+1}\left(k_{r} r\right) k_{r} \mathrm{~d} k_{r}+k_{0}^{2} \exp \left(-i \hat{k}_{z}^{i} z_{s}\right) \hat{b}_{n}^{H, i} J_{n+1}\left(k_{r}^{i} r\right)=\int_{0}^{\infty} \exp \left(-i k_{z} z_{s}\right) b_{n}^{H,-}\left(k_{r}\right) \mathbb{Z}_{n+1}\left(k_{r} r\right) k_{r} \mathrm{~d} k_{r} .
\end{aligned}
$$

form field maps that exhibit higher-order symmetry, which behave as $\sin (n \theta)$ or $\cos (n \theta)$. For $n=2$ the field map is doubly periodic with respect to $\theta$; for $n=3$ the map is triply periodic, etc. Whereas this is interesting from a theoretical point of view, we do not go further in detail, because these solutions (as well as for $n=0$ ) cannot be excited with a linearly polarized normally incident wave that is singly periodic in $\theta$ (Appendix A) and that is the subject of our numerical study, which follows.

\section{Perturbation Analysis of Surface-Wave Excitation Owing to Surface Defects}

Let us now consider a metal-dielectric interface supporting a plasmon surface wave that is slightly perturbed with a perturbation geometry that is independent of $\theta$. Let us assume that the surface equation is expressed in the form

$$
z_{S}=h f(r),
$$

where $h$ is a small parameter that represents the height of the perturbation and has a dimension of length. If $h \rightarrow 0$, we can assume that the boundary conditions require the continuity of $E_{ \pm, n}\left(r, z_{S}\right)$. Because of surface perturbation, the component of the wave vector in the $(x, y)$ plane is no longer preserved, and an incident wave characterized by a single value of $k_{r}=k_{r}^{i}$ of the form

$$
E_{ \pm, n}^{i}(r, z)=b_{ \pm, n}^{i}\left(k_{r}^{i}\right) \exp \left(-i \hat{k}_{z}^{i} z\right) J_{n \pm 1}\left(k_{r}^{i} r\right)
$$

will generate a continuum of coupled components with $0 \leq k_{r}<\infty$. In Eq. (27) it is necessary to use the first-kind Bessel function, which is the only nondiverging Bessel function for any value of $r$. From the general form given in Eq. (6), one can conclude that
Within the first-order approximation in $h$, the exponential terms become

$$
\exp \left( \pm i k_{z} z_{S}\right) \approx 1 \pm i h k_{z} f(r) .
$$

The zeroth-order terms correspond exactly to Eq. (16) for $b_{n}^{H}\left(k_{r}^{i}\right)$, which are the only field components that are present when $h=0$. The other components, characterized by $k_{r} \neq k_{r}^{i}$, are of higher order in $h$. We can further simplify the first-order approximation of Eqs. (29) by multiplying by $\mathbb{Z}\left(k_{r}^{\prime} r\right) r$ and integrating with respect to $r$ :

$$
\begin{gathered}
\frac{\hat{k}_{z}^{\prime}}{\hat{\varepsilon}} \hat{b}_{n}^{H,+}\left(k_{r}^{\prime}\right)+i \frac{\hat{k}_{z}^{i}}{\hat{\varepsilon}} \hat{b}_{n}^{H, i} F_{n+1}\left(k_{r}^{\prime}, k_{r}^{i}\right)=-\frac{k_{z}^{\prime}}{\varepsilon} b_{n}^{H,-}\left(k_{r}^{\prime}\right), \\
\hat{b}_{n}^{H,+}\left(k_{r}^{\prime}\right)-i \hat{b}_{n}^{H, i} F_{n+1}\left(k_{r}^{\prime}, k_{r}^{i}\right)=b_{n}^{H,-}\left(k_{r}^{\prime}\right),
\end{gathered}
$$

with coupling coefficient $F_{n+1}\left(k_{r}, k_{r}^{i}\right)$ equal to

$$
F_{n+1}\left(k_{r}, k_{r}^{i}\right)=k_{0}^{2} \hat{k}_{z}^{i} h \int_{0}^{\infty} f(r) \mathbb{Z}_{n+1}\left(k_{r} r\right) J_{n+1}\left(k_{r}^{i} r\right) r \mathrm{~d} r .
$$

On comparison of Eq. (31) with Eq. (16), it is evident that the diffracted-field amplitudes are expressed through the incident-field amplitude with similar Fresnel coefficients, multiplied by the coupling integral, Eqs. (32):

$$
\begin{aligned}
& \hat{b}_{n}^{H,+}\left(k_{r}\right)=-i F_{n+1}\left(k_{r}, k_{r}^{i}\right) \frac{\hat{k}_{z}^{i} / \hat{\varepsilon}-k_{z} / \varepsilon}{\hat{k}_{z} / \hat{\varepsilon}+k_{z} / \varepsilon} \hat{b}_{n}^{H, i}, \\
& b_{n}^{H,-}\left(k_{r}\right)=i F_{n+1}\left(k_{r}, k_{r}^{i}\right) \frac{\hat{k}_{z}^{i} / \hat{\varepsilon}+k_{z} / \varepsilon}{\hat{k}_{z} / \hat{\varepsilon}+k_{z} / \varepsilon} \hat{b}_{n}^{H, i} .
\end{aligned}
$$




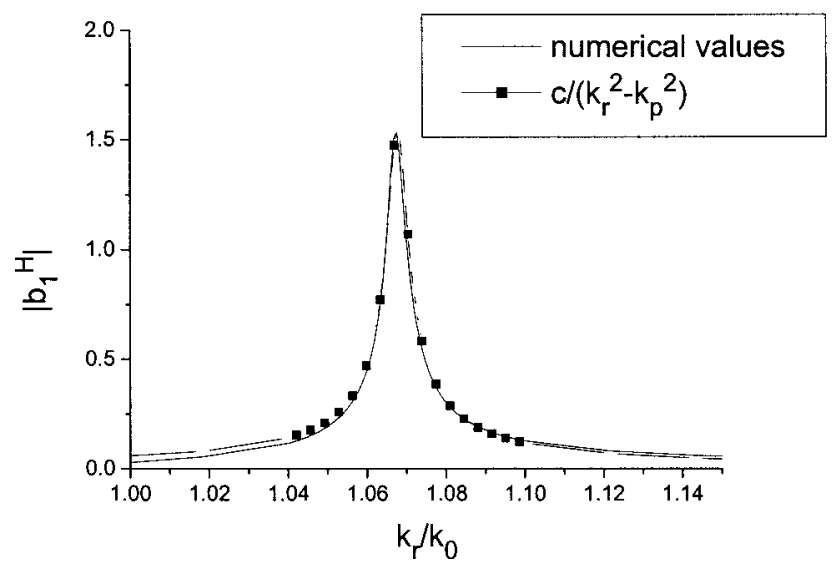

Fig. 3. Comparison of the computed $k_{r}$ dependence of $\left|\hat{b}_{1}^{H,+}\right|$ and its approximation given by the phenomenological formula [relation (34)] in the vicinity of the plasmon propagation constant for a silver screen with $50 \mathrm{~nm}$ radius of aperture and $\lambda=500 \mathrm{~nm}$.

It has to be stressed that these expressions are valid only in a first-order approximation in $h$. However, they permit us to draw three important conclusions: First, Eqs. (33) have the same poles as Eqs. (18), and thus the condition for the existence of surface plasmons given for a flat interface by Eq. (19) remains the same for a perturbed surface. Second, to maximize certain diffracted components by a suitable choice of the surface perturbation it is necessary to maximize coupling integral $F_{n+1}\left(k_{r}, k_{r}^{i}\right)$. Third, for values of $k_{r}$ close to the pole $k_{r}^{p}$, the field amplitudes can be expressed in series of $k_{r}-k_{r}^{p}$. However, because according to Eq. (11) $k_{z}=k_{z}\left(k_{r}^{2}\right)$, the dependence becomes

$$
\begin{aligned}
\hat{b}_{n}^{H,+}\left(k_{r}\right), b_{n}^{H,-}\left(k_{r}\right) & \sim \frac{1}{\hat{k}_{z} / \hat{\varepsilon}+k_{z} / \varepsilon} F_{n+1}\left(k_{r}, k_{r}^{i}\right) \hat{b}_{n}^{H, i} \\
& \approx \frac{C}{k_{r}^{2}-\left(k_{r}^{p}\right)^{2}} F_{n+1}\left(k_{r}, k_{r}^{i}\right) \hat{b}_{n}^{H, i} .
\end{aligned}
$$

The validity of this formula is illustrated in Fig. 3, where the $k_{r}$ dependence of $\hat{b}_{1}^{H,+}$, expanded on the basis of $J_{1}\left(k_{r} r\right),{ }^{6}$ is represented for linearly polarized light normally incident upon an air-silver interface, where the silver film is pierced with a $50 \mathrm{~nm}$ radius hole and the wavelength is $\lambda=500 \mathrm{~nm}$. As can be observed, in the vicinity of the pole the behavior is clearly expressed by relation (34), which is known in grating theories as a phenomenological formula.

\section{Optimization of Surface Perturbation}

Let us go back to the second conclusion of Section 4 and try to optimize the coupling integral to enhance the excitation of the plasmon surface wave. For a given perturbation form, the coupling is assumed to increase with the corrugation amplitude, at least up to certain values, because the linear dependence in
Eqs. (32) is limited to the first-order approximation in $h$ that is used. Higher-order terms will change this linear dependence, and they are automatically taken into account in the numerical result. In what follows, we limit ourselves to normally incident linearly polarized monochromatic light containing a single $k_{r}$ component, $k_{r}^{i}=0$. The coupling integral becomes

$$
F_{0}\left(k_{r}, k_{r}^{i}\right)=k_{0}^{2} \hat{k}_{z}^{i} h \int_{0}^{\infty} f(r) \mathbb{Z}_{0}\left(k_{r} r\right) r \mathrm{~d} r .
$$

It is thus evident that the optimal surface profile that presents the maximum coupling between the normally incident wave and the $k_{r}$ diffracted component will be obtained when the profile is represented by the zeroth-order Bessel function:

$$
f(r)=J_{0}\left(k_{r}^{\prime} r\right) \Rightarrow F_{0}\left(k_{r}, k_{r}^{i}\right)=k_{0}^{2} k_{z}^{i} h \frac{\delta\left(k_{r}-k_{r}^{\prime}\right)}{k_{r}^{\prime}} .
$$

The coupling is then infinitely strong. However, higher-order diffraction limits the coupling because of the radiation, as reciprocity requires that the coupling between the incident and the $k_{r}$ components be equal to the inverse coupling. Indeed, the coupling integral is symmetric with respect to $k_{r}$ and $k_{r}^{i}$.

Further on in this section we analyze numerically such strong singular coupling predicted by expressions (36), but it is quite difficult to fabricate this structure, so we deal first with more realistic lamellar-form corrugation.

\section{A. Single Aperture}

The validity of the approach is tested first on a single circular hole without surface corrugation. The plasmon wave is excited at the aperture and propagates outward; thus its field is expressed in the form $\mathbf{H}_{n}^{+}\left(k_{r}^{p} r\right)$ rather than in the standing-wave form, $J_{n}\left(k_{r}^{p} r\right)$. There is no contradiction of the fact that in relation (34) we used the basis of $J_{1}\left(k_{r} r\right)$ because of the relations between Bessel functions of different kinds. In particular, there is the following link between $J_{1}$ and $\mathbf{H}_{1}^{+}$for complex values of $k_{r}^{p}$ :

$$
\int_{0}^{\infty} J_{1}\left(k_{r} r\right) \frac{k_{r} \mathrm{~d} k_{r}}{k_{r}^{2}-\left(k_{r}^{p}\right)^{2}}=i \frac{\pi}{2} \mathbf{H}_{1}^{+}\left(k_{r}^{p} r\right) .
$$

Thus, by using relation (34) and working with a $J_{1}$ basis with real values of $k_{r}$, one can obtain an outgoing propagating solution with complex propagating constant $k_{r}^{p}$ corresponding to a pole of Eqs. (18). This fact can be observed from Fig. 4, where the modulus of $E_{z}$ exactly follows the function $\mathbf{H}_{1}^{+}\left(k_{r}^{p} r\right)$ outside the aperture.

However, inside the hole, the field components must be represented in the basis of $J_{n}$, which does not diverge at origin. Then the coupling integral is extended from 0 to $R$ and takes a simple form: 


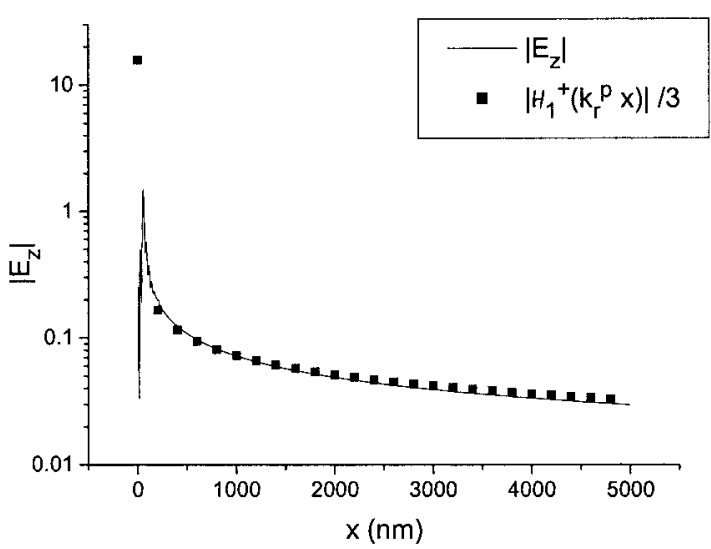

Fig. 4. Field map of a surface plasmon wave excited by a circular aperture of $50 \mathrm{~nm}$ radius in a silver film by an $x$-polarized normally incident plane wave with wavelength equal to $500 \mathrm{~nm}$. Rigorous results, solid curve; corresponding Hankel function, squares.

$$
F_{0}\left(k_{r}^{p}, 0\right)=k_{0}^{2} \hat{k}_{z}^{i} h \int_{0}^{R} J_{0}\left(k_{r}^{p} r\right) r \mathrm{~d} r=k_{0}^{2} \hat{k}_{z}^{i} h \frac{R}{k_{r}^{p}} J_{1}\left(k_{r} R\right) .
$$

At $\lambda=500 \mathrm{~nm}$, the plasmon propagation constant on an air-silver interface is $\alpha^{p}=k_{r}^{p} / k_{0} \approx 1.068$. The incident wave has no $z$ component of the field, and the dependence of $\left|E_{z}\right|$ along the surface is determined mainly by the plasmon surface wave. Its value far from the aperture $(x=4 \mu \mathrm{m})$ is plotted in Fig. 5 as a function of the aperture radius, together with the dependence on the perturbative approach given by Eq. (38). One can observe a very good correspondence between the rigorous numerical results and the perturbation analysis. Figure 6 presents a comparison of the two sets of results, given as a function of the wavelength for a fixed aperture dimension, $R$ $=50 \mathrm{~nm}$. Here it is necessary to rigorously take into account the dispersion of $\alpha^{p}$.

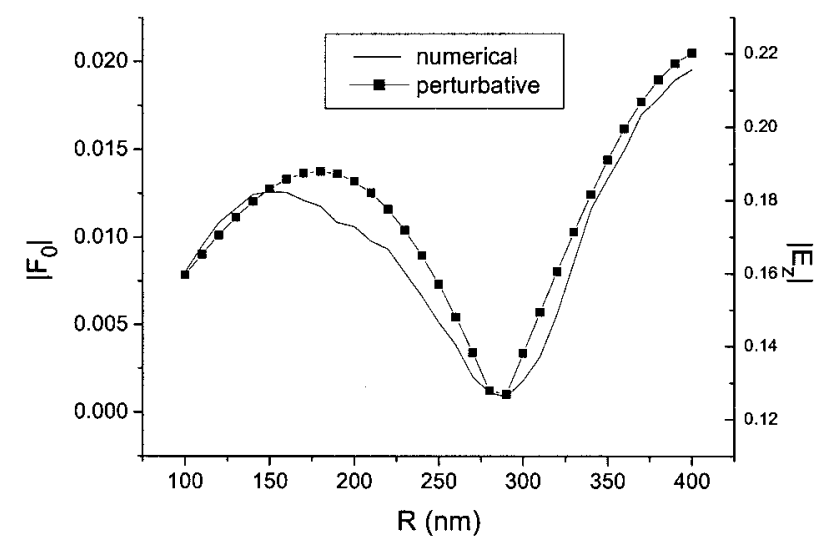

Fig. 5. Comparison of values of $\left|E_{z}\right|$ at point $(4 \mu \mathrm{m}, 0,0)$ computed from the rigorous theory and from the perturbative approach. Aperture radius $R$ is varied; other parameters are the same as in Fig. 4.

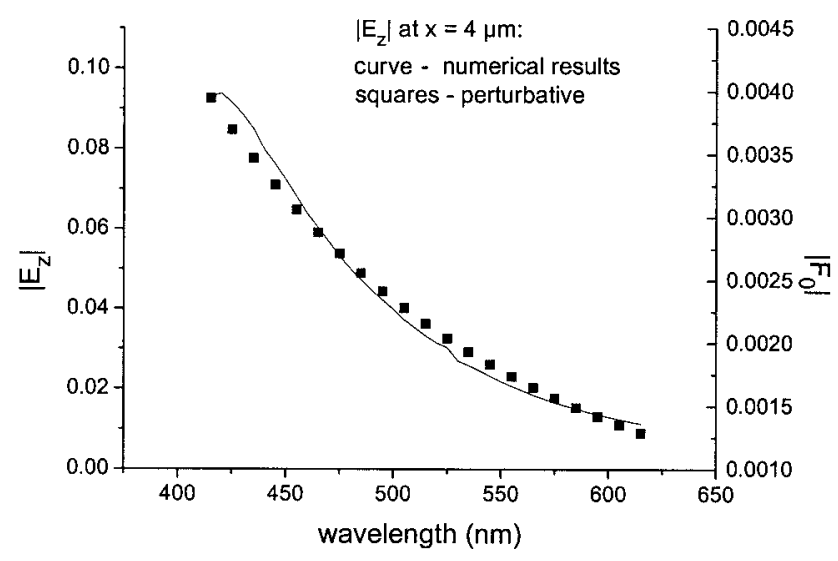

Fig. 6. Same as in Fig. 5 but with the wavelength varied and $R$ $=50 \mathrm{~nm}$.

\section{B. Lamellar Corrugation}

In analogy with the diffraction grating behavior, one expects to resonantly enhance the surface wave by using a suitably positioned corrugation to excite the surface wave in phase along a surface much larger than the single aperture. As is obvious from the analysis of expressions (36), singular resonant excitation can be expected if the corrugation follows the Besselfunction quasi-periodicity rather than using equidistantly corrugated channels. To investigate this assumption it is necessary first to calculate the coupling integral for the multichannel corrugation that was schematically presented in Fig. 1 . The discontinuity of the radial electric field components across the channel walls requires that the coupling integral take different forms in the channels and between them:

$$
\begin{aligned}
F_{0}\left(k_{r}^{p}, 0\right)= & k_{0}^{2}\left[\hat{k}_{z}^{i} h \int_{0}^{R_{1}} J_{0}\left(k_{r}^{p} r\right) r \mathrm{~d} r\right. \\
& +k_{z}^{i} h \int_{R_{1}}^{R_{2}} J_{0}\left(k_{r}^{p} r\right) r \mathrm{~d} r \\
& \left.+\hat{k}_{z}^{i} h \int_{R_{2}}^{R_{3}} J_{0}\left(k_{r}^{p} r\right) r \mathrm{~d} r+\ldots\right] \\
= & k_{0}^{2}\left(\hat{k}_{z}^{i}-k_{z}^{i}\right) \frac{h}{k_{r}^{p}} \sum_{j=1}^{j_{\max }}(-1)^{j} R_{j} J_{1}\left(k_{r}^{p} R_{j}\right) .
\end{aligned}
$$

As observed further, sometimes it is better to use an alternative form of this expression because the radial component of the plasmon field is represented by the expression $\mathbb{Z}_{0}-\mathbb{Z}_{2}$ [Eqs. (22)] rather than simply by $\mathbb{Z}_{0}$. Another form of Eq. (39) is then easily obtained:

$$
\begin{aligned}
F_{0}\left(k_{r}^{p}, 0\right)= & k_{0}^{2}\left(\hat{k}_{z}^{i}-k_{z}^{i}\right) \frac{h}{k_{r}^{p}} \sum_{j=1}^{j_{\max }}(-1)^{j} R_{j} \\
& \times\left[J_{1}\left(k_{r}^{p} R_{j}\right)-J_{3}\left(k_{r}^{p} R_{j}\right)\right] .
\end{aligned}
$$




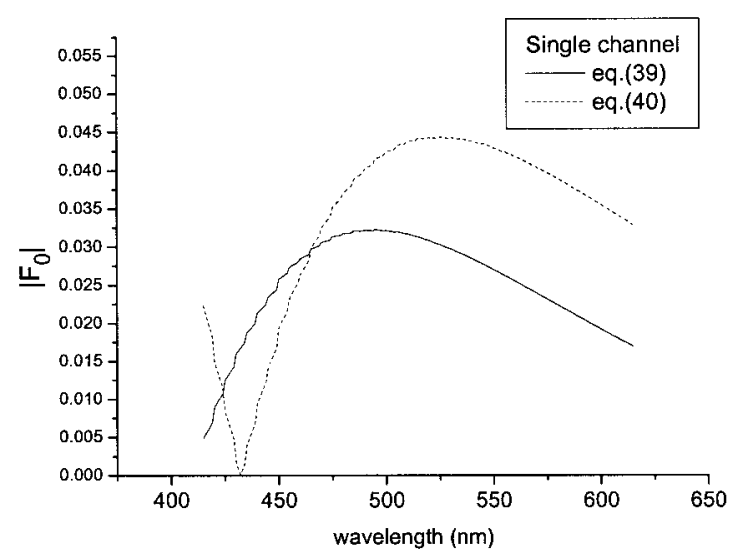

(a)

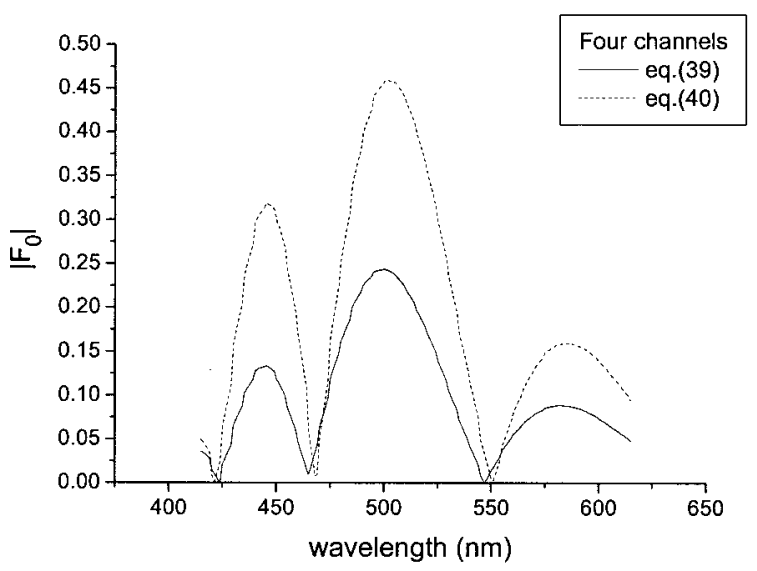

(b)

Fig. 7. Comparison of two expressions for the coupling integral given in Eqs. (39) and (40) as a function of wavelength for a Besselfunction-type corrugation with radii given in Table 1 and situated about a $50 \mathrm{~nm}$ radius aperture.

The two expressions differ significantly for a singlechannel corrugation $\left[j_{\max }=3\right.$; Fig. $\left.7(\mathrm{a})\right]$, and their difference is reduced when the number of channels increases, as shown in Fig. 7(b). As is evident from Eq. (39), to maximize the coupling integral for a given value of the corrugation depth it is necessary to introduce the channel walls (i.e., $R_{j}$ ) consecutively, at the maxima and minima of the function $r J_{1}\left(k_{r}^{p} r\right)$. Then, increasing the total number of channels will increase the coupling integral and change its spectral behavior significantly (Fig. 8), the maxima becoming higher and narrower. The curves are drawn with values of $R_{j}$ optimized to obtain the maximum of the coupling integral at $\lambda=500 \mathrm{~nm}$. With the value of the plasmon propagating constant equal to 1.068 , the corresponding optimal values for $R_{j}$ are as given in Table 1 . We keep the same the hole radius, $R_{1}$ $=50 \mathrm{~nm}$. These results explain why in Fig. 2 the spectral maximum positions differs significantly from $500 \mathrm{~nm}$.

The expectations from the approximate formulas are fully confirmed by rigorous numerical results. As we observe below, for a given configuration the maximum field enhancement is obtained for shallow cor-

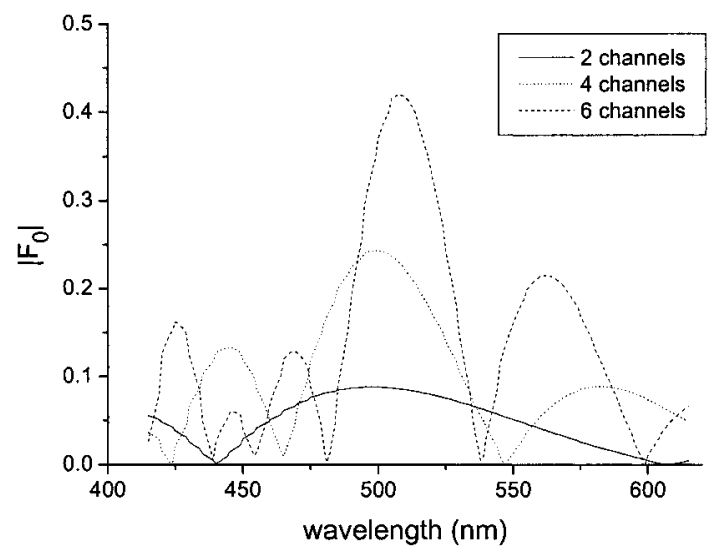

Fig. 8. Spectral dependence of the coupling integral given by Eq. (39) and its evolution when the number of channels is increased for the same structure as in Fig. 7.

rugation depth, of the order of 30-50 nm (Fig. 9, fourchannel corrugation). This is explained by the fact that deeper corrugations lead to stronger radiation of the plasmon and perturb its propagation by cutting the surface. Similar behavior exists when a plasmon surface wave is excited on the surface of sinusoidal or lamellar metallic diffraction gratings. In what follows, we have chosen $h=30 \mathrm{~nm}$.

Figure 10 shows the dependence of the field enhancement on the number of corrugation channels, compared with a single-aperture configuration. The plasmon field is calculated at $x=4 \mu \mathrm{m}$ away from the corrugation, because at the channel walls one observes strong local field fluctuations. Both the electric field and the total transmission by the aperture are strongly enhanced, as expected from Fig. 8. The spectral behavior of the plasmon amplitude is quite well predicted by Eq. (40) or (39) for one or four channels, respectively, as seen from Fig. 11, although a small spectral shift to a longer wavelength is observed for the rigorous results.

Figure 12 presents the spectral dependence of the plasmon field for a single corrugated channel with walls situated at 235 and $470 \mathrm{~nm}$, a part of the equidistant structure discussed in Fig. 2. A sharp minimum is observed close to $480 \mathrm{~nm}$, the spectral position close to which one would expect a maximum, judging from the one-dimensional grating theory $(500 \mathrm{~nm})$. However, this geometry outcome is not surprising because the correct phenomenological formula from Eq. (40) matches the numerical results in both Figs. 2 and 12. The field amplitude is similar in values when an equidistant corrugation is used compared with the optimized corrugation from the table above; however, the spectral position of the maximum is significantly changed.

\section{Bessel-Function Corrugation}

As was already discussed, the optimal structure is assumed to have a geometry that varies according to the zeroth-order Bessel function [expressions (36)]. Because of numerical problems, we are not yet able to 
Table 1. Optimum Values of $\boldsymbol{R}_{j}$

\begin{tabular}{lccccrrrrrrrrrr}
\hline$j$ & 2 & 3 & 4 & 5 & 6 & 7 & 8 & 9 & 10 & 11 & 12 & 13 & 14 & 15 \\
$R_{j}(\mathrm{~nm})$ & 179.4 & 411.7 & 645.4 & 879.4 & 1113 & 1347.8 & 1582 & 1816 & 2051 & 2285.2 & 2517 & 2755.2 & 2985 & 3222.2 \\
\hline
\end{tabular}

model rigorously a continuous-profile surface corrugation in cylindrical geometry. However, the code can deal with multilevel corrugation; thus we investigated a surface that has the profile given in Fig. 13. In fact, the figure presents a staircase approximation of the zeroth-order Bessel function, limited in the $r$ direction. The phenomenological curves calculated from Eq. (39) for two values of $R_{\max }$ are shown in Fig. 14. If the corrugation as well as the incident wave were extended to infinity, the response would be high and narrow, as we observe in Subsection 5.D below. However, this geometry is of little practical interest because the energy density of the incident wave decreases with the beam width. Anyway, the main difference between the curves in Fig. 14 compared with the curves in Fig. 8 is the lower value of the secondary

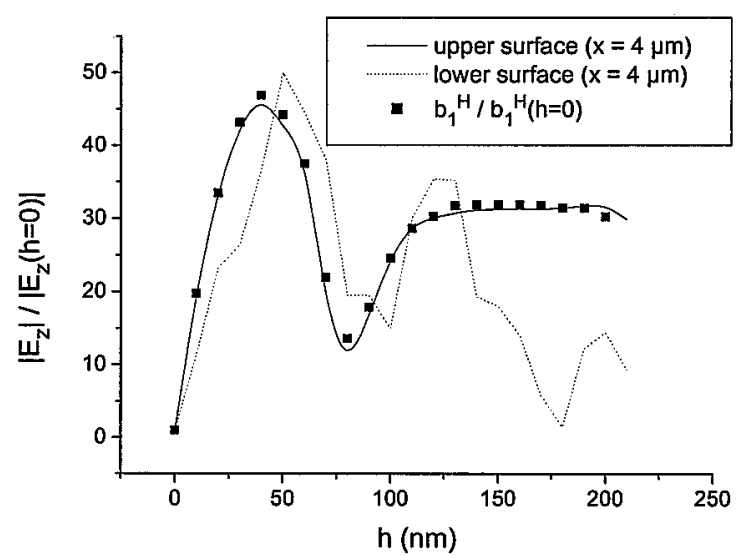

Fig. 9. Field enhancement as a function of channel depth for a four-channel structure surrounding a single $50 \mathrm{~nm}$ radius aperture at $\lambda=500 \mathrm{~nm}$. Field calculated on the upper and lower film surfaces compared with the enhancement of $\left|\hat{b}_{1}^{H,+}\right|$.

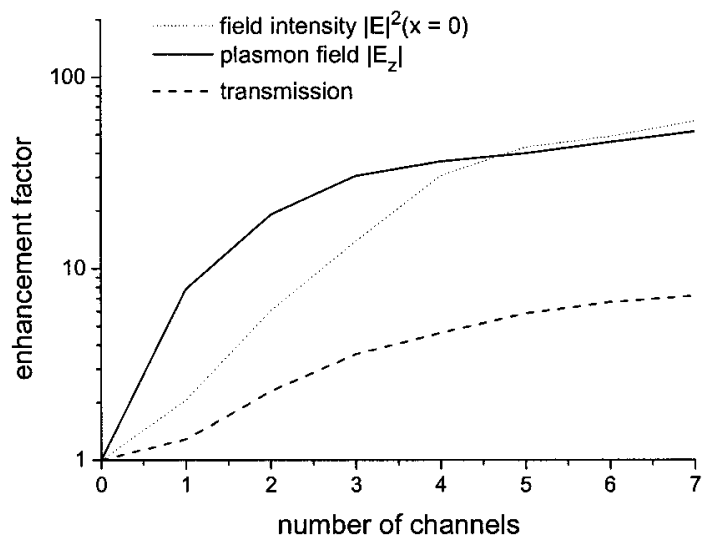

Fig. 10. Near-, transmitted-, and plasmon-field enhancement as a function of the number of channels for $h=50 \mathrm{~nm}$ and $\lambda$ $=500 \mathrm{~nm}$. maxima of the former, which is a natural consequence of the smoother profile.

The numerical results (Fig. 15) fully confirm the phenomenological predictions concerning the spectral width and position of the main maximum and the reduction of the secondary maxima. Again, a relatively small shift toward a longer wavelength is observed in the rigorous results.

\section{Phase Modulation}

The optimal corrugation given by expressions (36) is quite difficult to fabricate. However, instead of a surface corrugation, it is possible to use a phase modulation of an adjacent dielectric layer, in analogy with the phase gratings used for mode coupling in integrated optics. Such a phase structure can be manufactured by a holographic technique. Let us assume that it consists of a plane dielectric layer deposited

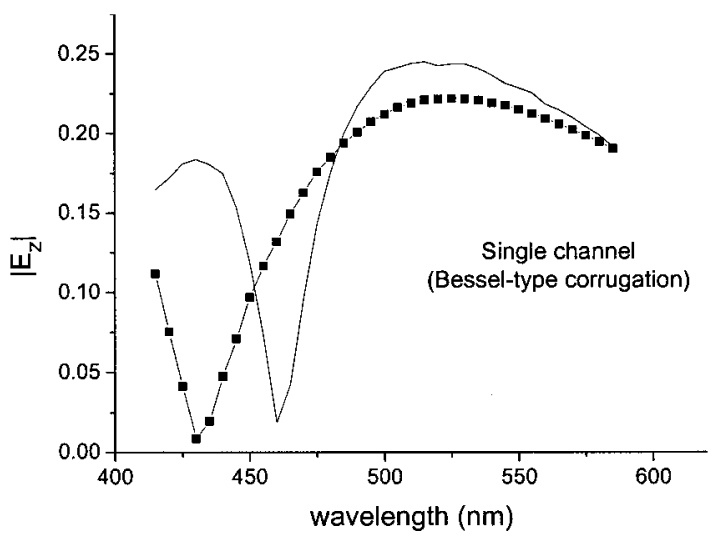

(a)

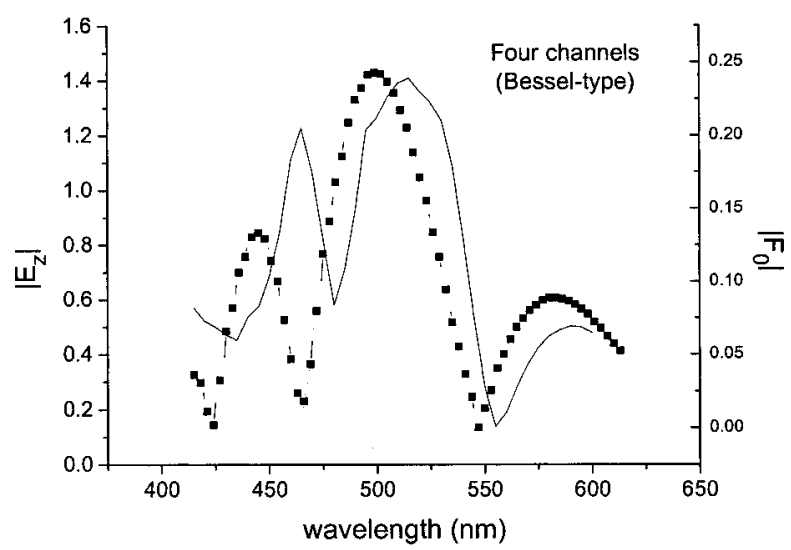

(b)

Fig. 11. Spectral dependence of $\left|E_{z}\right|$ at point $(4 \mu \mathrm{m}, 0,0)$ computed from the rigorous theory (solid curves) and the perturbative approach (squares): (a) single channel and Eq. (40), (b) four channels and Eq. (39). Corrugation radii follow the values in Table 1. 


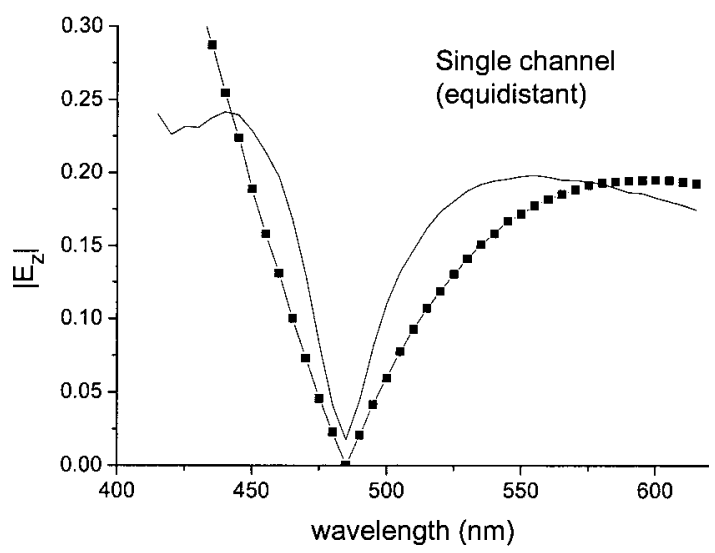

Fig. 12. Same as in Fig. 11(a) but for a single channel with $R_{2}$ $=235 \mathrm{~nm}$ and $R_{3}=470 \mathrm{~nm}$.

onto a metallic surface and characterized by a radial dependence of its refractive index. Applying the same procedure as when we went from Eq. (7) to Eq. (9), we obtain a coupled-wave equation:

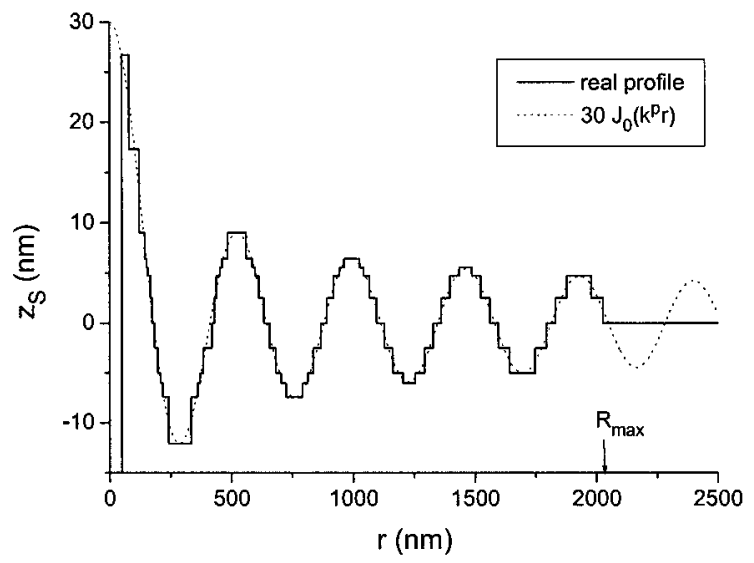

Fig. 13. Staircase approximation of smoothly varying surface modulation described by the zeroth-order Bessel function.

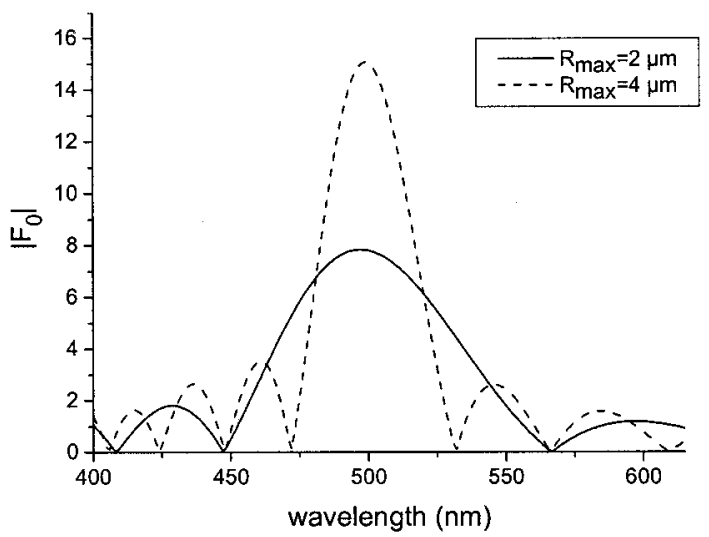

Fig. 14. Spectral dependence of the coupling integral obtained by use of two values of radius $R_{\max }$ of the modulated region.

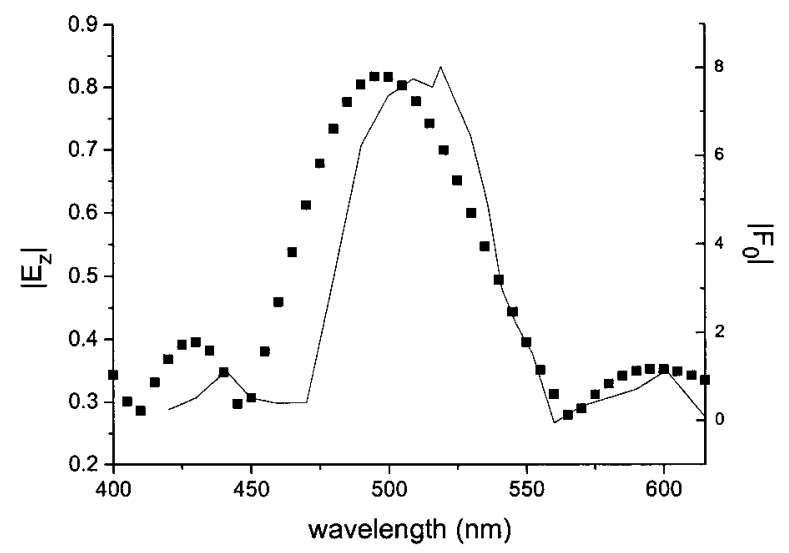

Fig. 15. Spectral dependence of the rigorous (solid curve) and perturbative (squares) values of $\left|E_{z}\right|$ at point $(4 \mu \mathrm{m}, 0,0)$ for the structure presented in Fig. 13.

$$
\begin{aligned}
& k_{r}^{2} e_{ \pm, n}\left(k_{r}, z\right)-\frac{\partial^{2}}{\partial z^{2}} e_{ \pm, n}\left(k_{r}, z\right) \\
& \quad=\omega^{2} \mu_{0} \int_{0}^{\infty} \varepsilon(r) \mathbb{Z}_{n \pm 1}\left(k_{r} r\right) J_{n \pm 1}\left(k_{r}^{i} r\right) r \mathrm{~d} r e_{ \pm, n}\left(k_{r}, z\right) .
\end{aligned}
$$

The coupling integral that appears in Eq. (41) is responsible for the diffraction effects and has almost the same form as Eqs. (32), as can easily be understood because one can consider a lamellar structure as a phase grating with large contrast.

As has already been discussed with respect to expression (36), the strongest excitation can be expected if the perturbation follows the zeroth-order Bessel function. The response is expected to be dominated by a Dirac distribution, infinitely narrow and high. This is so because both the modulated region and the incident wave extend to infinity in direct space, which results in a single-frequency excitation in $k_{r}$ space. This is an exact analogy of a diffraction order of a classic diffraction grating being infinitely narrow spectrally and angularly if the grating has infinite dimensions and is illuminated by a perfect plane wave. Whereas this situation represents an academic abstraction, its analysis enables us to understand the underlying physics better. Anyway, a real finite system or a finite-width incident beam or both are assumed to exhibit responses much closer to those shown in Figs. 14 and 15.

Taking into account the above-mentioned remarks, let us analyze a system consisting of a single circular aperture in a $200 \mathrm{~nm}$ thick silver screen covered by a thin high-index dielectric layer with its dielectric permittivity modulated according to the following formula:

$$
\varepsilon(r)=\varepsilon_{d}+\Delta \varepsilon J_{0}\left(k_{r}^{\mathrm{mod}} r\right),
$$

where $k_{r}^{\text {mod }}$ is a constant chosen to be equal to the propagation constant of the plasmon surface wave 


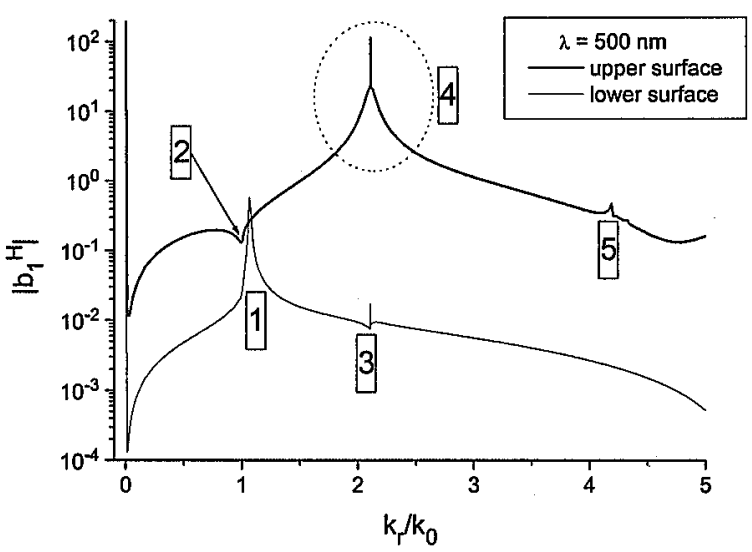

Fig. 16. Dependence of $\left|\hat{b}_{1}^{H,+}\right|$ at $z=0$ (upper metallic interface) and of $\left|b_{1}^{H,-}\right|$ at $z=-200 \mathrm{~nm}$ (lower surface) on the values of $k_{r} / k_{0}$ for a $50 \mathrm{~nm}$ radius aperture in a silver film covered with a $50 \mathrm{~nm}$ thick dielectric layer with permittivity modulated according to Eq. (42), $\lambda=500 \mathrm{~nm}, \varepsilon_{d} / \varepsilon_{0}=3.5, \Delta \varepsilon / \varepsilon_{0}=0.1$, and $k_{r}^{\text {mod }}$ $=0.02649 \mathrm{~nm}^{-1}$.

propagating along the metal-dielectric interface for a given wavelength. If it is thick enough, the dielectric cover layer can support waveguide modes. To prevent this from occurring it is necessary to have a sufficiently thin layer. In addition, the layer's existence will modify the plasmon propagation constant, as can be observed from Fig. 16, where the $k_{r}$ decomposition of the $z$ electric field component is represented for the upper and the lower metallic surfaces, similarly to Fig. 3. There are several features, indicated in the figure by numbers inside rectangles:

1. There is an anomaly that is due to the plasmon excitation at the lower metal-air interface. It is similar to that in Fig. 3, except for the appearance of a small feature with number 3 . The amplitude of the field at this interface is naturally lower than on the upper boundary.

2 . The existence of this surface wave is also manifested in the field at the upper boundary, owing to the coupling through the circular aperture.

3. The constant $k_{r}^{\bmod }=0.02649 \mathrm{~nm}^{-1}$ in the mod-

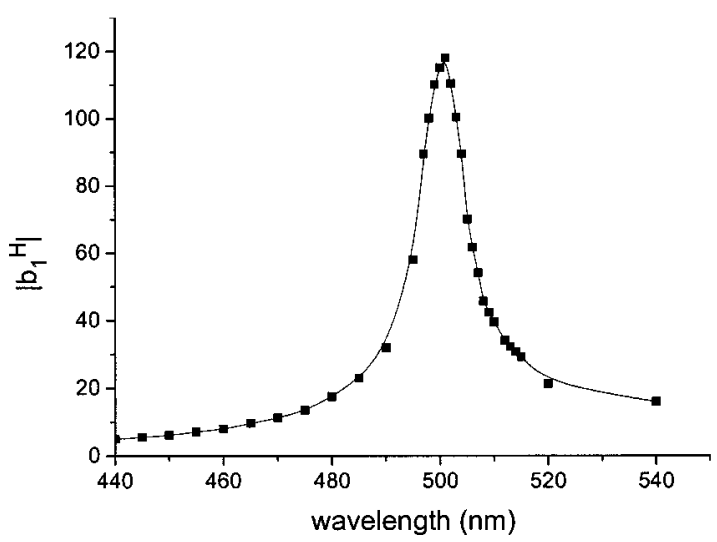

Fig. 17. Spectral dependence of $\left|\hat{b}_{1}^{H,+}\right|$ for the structure described in Fig. 16.

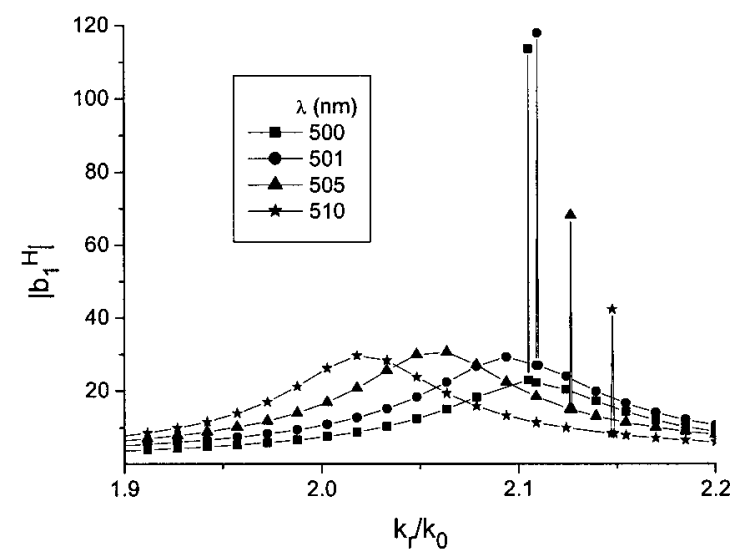

Fig. 18. $\left|\hat{b}_{1}^{H,+}\right|$ as a function of $k_{r} / k_{0}$ in the vicinity of plasmon excitation on the interface metal-modulated dielectric layer for the structure described in Fig. 16 and presented for four wavelengths.

ulation function is chosen to correspond to the upper-boundary plasmon propagation constant at $\lambda=500 \mathrm{~nm}$. The effect of the modulation is quite strong at the upper metallic boundary but can also be observed at the lower boundary.

4. Feature 4 consists of a relatively wide maximum representing the upper-surface plasmon excitation by the aperture, similar to Fig. 3 . The width is determined by attenuation constant $\gamma^{p}=\operatorname{Im}\left(\alpha^{p}\right)$ of the plasmon, equal to $\sim 0.04$ at $\lambda=500 \mathrm{~nm}$. In addition to this broad peak, one can observe a vertical feature with one-pixel width; the pixel is equal to a single step in the numerical discretization along the $k_{r}$ axis. This feature is due to the effect of the modulation of the dielectric layer and is analyzed in further detail below.

5. Feature 4 is translated along the $k_{r}$ axis with a
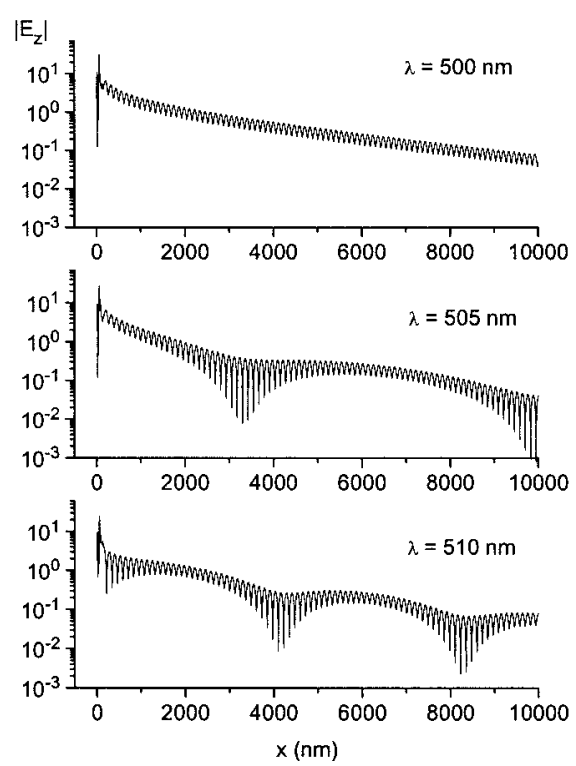

Fig. 19. Spatial variation of the plasmon field for three wavelengths for the structure given in Fig. 16. 
period equal to $k_{r}^{\text {mod }}$, representing a higher-order coupling.

In what follows, we turn our attention to feature 4 in particular, as it represents the resonant plasmon excitation discussed in this paper. The spectral behavior of the amplitude of the electric field of the plasmon surface wave is given in Fig. 17. As expected, the curve is much narrower than for a limited-region modulation (Figs. 14 and 15) but not infinitely thin, as expected from expressions (36). This is so because the response depends both on the coupling integral and on the plasmon spectral curve, not to forget about its dispersion characteristics. All these arguments are illustrated in $k_{r}$ space in Fig. 18. When the wavelength is varied, the constant of modulation $k_{r}^{\bmod }$ does not move in $k_{r}$ space, although there is a slight drift along the normalized $k_{r}-k_{0}$ axis. This representation was chosen to distinguish the variation of the peak amplitudes with wavelength. As implied by relation (34), the modulation of the refractive index induces (through the coupling integral) variations of the electromagnetic field that has the same modulation characteristics, i.e., has a single frequency representation in $k$ space. From the point of view of plasmon excitation, this field modulation serves as an external resonant source.

The plasmon propagation constant varies with the wavelength. This variation is due to the dispersion of silver and, mainly, to the geometric dispersion of the dielectric layer; the modification of the plasmon that is due to its presence is strongly influenced by wavelength, as can be observed in Fig. 18 as a relatively rapid displacement of the broad maximum toward smaller $k_{r} / k_{0}$ values when the wavelength increases. Depending on the frequency mismatch between the external $\left(k_{r}^{\bmod }\right)$ and the proper $\left[k_{r}^{p}(\lambda)\right]$ metallicdielectric interface resonance, the resonance excitation could be stronger or weaker. Its spectral width is determined mainly by the wider resonance curve; this explains why spectral curve width $W$ in Fig. 17 is directly linked to the attenuation constant of the plasmon: $W \sim \lambda \gamma^{p} \approx 20 \mathrm{~nm}$.
Another direct consequence of the existence of two resonances (external, which is due to the layer index modulation, and proper, which is the plasmon surface wave) is that the diffracted field no longer represents an outgoing (toward $r \rightarrow \infty$ ) wave, as presented in Fig. 4 , but is rather a combination of two coupled waves: the outgoing plasmon field and a standing wave excited by the modulation of the dielectric layer, a modulation that extends to infinity. Because of the coupling, the field exhibits a rapidly varying character [period of variation determined by the mean frequency $\left.\left(k_{r}^{\bmod }+k_{r}^{p}\right) / 2\right]$ and large-period beating with frequency equal to $\left|k_{r}^{\bmod }-k_{r}^{p}(\lambda)\right|$, as can be observed from Fig. 19 for the exact frequency match $(500 \mathrm{~nm}$, no beating) and for two other frequencies that produce smaller- or larger-period beating effects. Results not reproduced here show that rigorous calculations confirm that the plasmon excitation is a linear function of $\Delta \varepsilon$ in a large interval $\left(0 \leq \Delta \varepsilon \leq 2.5 \varepsilon_{0}\right)$, as predicted by expressions (36) and (42).

\section{Conclusions}

A rigorous electromagnetic study combined with perturbative analysis has shown that it is possible to optimize the optogeometrical parameters of structured circular apertures in metallic films for stronger excitation of surface plasmon waves and thus of the local field close to the surface by using simple rules based on knowledge of plasmon field peculiarities in cylindrical geometry. The optimal surface corrugation has to follow the Bessel functions rather than be equidistant. A lamellar cross-section corrugation with constant groove depth has to be structured according to the minima and maxima of the first-order Bessel function (Table 1), whereas a smoothly varying surface relief or refractive-index (phase) modulation has to follow the zeroth-order Bessel function [expression (36) or (42)].

\section{Appendix A. Maxwell's Equations in Cylindrical Coordinates}

Maxwell's equations written in cylindrical coordinates have the form

$$
\begin{aligned}
& \frac{1}{r} \frac{\partial E_{z}}{\partial \theta}-\frac{\partial E_{\theta}}{\partial z}=i \omega \mu_{0} H_{r}, \\
& \frac{\partial E_{r}}{\partial z}-\frac{\partial E_{z}}{\partial r}=i \omega \mu_{0} H_{\theta}, \\
& \frac{\partial E_{\theta}}{\partial r}+\frac{E_{\theta}}{r}-\frac{1}{r} \frac{\partial E_{r}}{\partial \theta}=i \omega \mu_{0} H_{z}, \\
& \frac{1}{r} \frac{\partial H_{z}}{\partial \theta}-\frac{\partial H_{\theta}}{\partial z}=-i \omega \varepsilon E_{r}, \\
& \frac{\partial H_{r}}{\partial z}-\frac{\partial H_{z}}{\partial r}=-i \omega \varepsilon E_{\theta}, \\
& \frac{\partial H_{\theta}}{\partial r}+\frac{H_{\theta}}{r}-\frac{1}{r} \frac{\partial H_{r}}{\partial \theta}=-i \omega \varepsilon E_{z},
\end{aligned}
$$

$$
\begin{aligned}
& \frac{\partial E_{\theta, n}}{\partial z}=\frac{i n}{r} E_{z, n}-i \omega \mu_{0} H_{r, n}, \\
& \frac{\partial E_{r, n}}{\partial z}=\frac{\partial E_{z, n}}{\partial r}+i \omega \mu_{0} H_{\theta, n}, \\
& \mathrm{i} \omega \mu_{0} H_{z, n}=\frac{\partial E_{\theta, n}}{\partial r}+\frac{E_{\theta, n}}{r}-\frac{i n}{r} E_{r, n}, \\
& \Rightarrow \\
& \frac{\partial H_{\theta, n}}{\partial z}=\frac{i n}{r} H_{z, n}+i \omega \varepsilon E_{r, n}, \\
& \frac{\partial H_{r, n}}{\partial z}=\frac{\partial H_{z, n}}{\partial r}-i \omega \varepsilon E_{\theta, n}, \\
& -i \omega \varepsilon E_{z, n}=\frac{\partial H_{\theta, n}}{\partial r}+\frac{H_{\theta, n}}{r}-\frac{i n}{r} H_{r, n},
\end{aligned}
$$


where the set at the right is written with the $\theta$ dependence in Eq. (1) taken explicitly into account. The third and the sixth of Eqs. (A1) can be simplified by use of Eqs. (4) and (6):

$$
\begin{aligned}
& i \omega \mu_{0} H_{z, n}=\int_{0}^{\infty}\left(e_{+, n}-e_{-, n}\right) \mathbb{Z}_{n}\left(k_{r} r\right) k_{r}^{2} \mathrm{~d} k_{r}, \\
& -i \omega \varepsilon E_{z, n}=\int_{0}^{\infty}\left(h_{+, n}-h_{-, n}\right) \mathbb{Z}_{n}\left(k_{r} r\right) k_{r}^{2} \mathrm{~d} k_{r} .
\end{aligned}
$$

Let us consider a homogeneous region where $\varepsilon$ is constant. Then Eqs. (A2) immediately lead to Eqs. (13). The derivatives of $E_{z, n}$ and $H_{z, n}$ with respect to $r$ in Eqs. (A1) can be explicitly obtained from the relation $\mathbb{Z}_{n}^{\prime} \pm n \mathbb{Z}_{n} / r= \pm \mathbb{Z}_{n \mp l}$, and Eqs. (A1) can be rewritten for the new field components $E_{ \pm, n}$ and $H_{ \pm, n}$. They can be further simplified by separate consideration of the two fundamental polarizations:

\section{A. TE Polarization}

For TE polarization the first two of Eqs. (A1) can be combined if one takes into account that $E_{z, n}=0$ :

$$
\begin{aligned}
\frac{\partial}{\partial z} E_{+, n} & =\omega \mu_{0} H_{+, n} \Rightarrow \pm i k_{z} b_{n}^{E, \pm}=\omega \mu_{0} b_{n}^{H, \pm}, \\
\frac{\partial}{\partial z} E_{-, n} & =-\omega \mu_{0} H_{-, n} \Rightarrow \pm i k_{z} c_{n}^{E, \pm}=-\omega \mu_{0} c_{n}^{H, \pm},
\end{aligned}
$$

a link used in Eqs. (14).

\section{B. TM Polarization}

For TM polarization the fourth and fifth of Eqs. (A1) can be combined if one takes into account that $H_{z, n}$ $=0$ :

$$
\begin{aligned}
& \frac{\partial}{\partial z} H_{+, n}=-\omega \varepsilon E_{+, n} \Rightarrow \pm i k_{z} b_{n}^{H, \pm}=-\omega \varepsilon b_{n}^{E, \pm}, \\
& \frac{\partial}{\partial z} H_{-, n}=\omega \varepsilon E_{-, n} \Rightarrow \pm i k_{z} c_{n}^{H, \pm}=\omega \varepsilon c_{n}^{E, \pm},
\end{aligned}
$$

a link used in Eqs. (15).

A linearly polarized normally incident plane wave has a simple expression. In the numerical calculations we use a monochromatic incident wave polarized in the $x$ direction. In cylindrical coordinates it has the following electric field components:

$$
E_{r}^{i}=E_{x}^{i} \cos \theta, E_{\theta}^{i}=-E_{x}^{i} \sin \theta, E_{z}^{i}=0,
$$

which shows that such a wave has only $n= \pm 1$ non-null Fourier harmonics.

The support of the EC-funded projects PHOREMOST (FP6/2003/IST/2-511616) is gratefully acknowledged. The content of this work is the sole responsibility of the authors.

\section{References}

1. T. W. Ebbesen, H. J. Lezec, H. F. Ghaemi, T. Thio, and P. A. Wolff, "Extraordinary optical transmission through subwavelength hole arrays," Nature 391, 667-669 (1998).

2. A. Moreau, G. Granet, F. I. Baida, and D. Van Labeke, "Light transmission by subwavelength square coaxial aperture arrays in metallic films," Opt. Express 11, 1131-1136 (2003).

3. F. J. Garcia-Vidal, L. Martin-Moreno, H. J. Lezec, and T. W. Ebbesen, "Focusing light with a single subwavelength aperture flanked by surface corrugations," Appl. Phys. Lett. 83, 4500-4502 (2003).

4. L. Martin-Moreno, F. J. Garcia-Vidal, H. J. Lezec, A. Degiron, and T. W. Ebbesen, "Theory of highly directional emission from a single subwavelength aperture surrounded by surface corrugations," Phys. Rev. Lett. 90, 167401 (2003).

5. R. Zakharian, M. Mansuripur, and J. V. Moloney, "Transmission of light through small elliptical apertures," Opt. Express 12, 2631-2648 (2004).

6. N. Bonod, E. Popov, and M. Nevière, "Differential theory of diffraction by finite cylindrical objects," J. Opt. Soc. Am. A 22, 481-490 (2005).

7. E. Popov, N. Bonod, M. Nevière, H. Rigneault, P.-F. Lenne, and P. Chaumet, "Surface plasmon excitation on a single subwavelength hole in a metallic sheet," Appl. Opt. 12, 2332-2337 (2005).

8. W. C. Chew and L. Gurel, "Reflection and transmission operators for strips or disks embedded in homogeneous and layered media," IEEE Trans. Microwave Theory Technol. 36, 14881497 (1988).

9. W. C. Chew, Waves and Fields in Inhomogeneous Media (Van Nostrand Reinhold, 1990).

10. V. A. Kosobukin, "Polarization and resonance effects in optical initiation of cylindrical surface-polaritons and periodic structures," Fizi. Tverd. Tela (Leningrad) 35, 884-898 (1993).

11. P. J. Valle, E. M. Ortiz, and J. M. Saiz, "Near field by subwavelength particles on metallic substrates with cylindrical surface plasmon excitation,” Opt. Commun. 137, 334-342 (1997). 